\title{
Tarifbindung und betriebliche Interes- senvertretung: Aktuelle Ergebnisse aus dem IAB-Betriebspanel 2011
}

In diesem Beitrag werden - wie schon in den letzten Jahren ${ }^{\bullet}$ - aktuelle Ergebnisse zur Tarifbindung und betrieblichen Interessenvertretung präsentiert. Die Befunde basieren auf Daten des Betriebspanels des Instituts für Arbeitsmarkt- und Berufsforschung (IAB-Betriebspanel), das als einzige repräsentative Datenquelle entsprechende Auskünfte in einer langen Zeitreihe für die Gesamtwirtschaft liefert.

PETER ELLGUTH, SUSANNE KOHAUT

\section{Einleitung}

Das deutsche System der Arbeitsbeziehungen wird durch die gesetzlichen Bestimmungen zur Tarifautonomie und betrieblichen Interessenvertretung durch Betriebsräte getragen. Überbetriebliche Branchen- oder Flächentarifverträge spielen eine wesentliche Rolle bei der Regelung von Arbeitsbedingungen wie auch bei der Lohnfindung. Sie werden meist für Regionen und Branchen ausgehandelt und sorgen dort für einheitliche Wettbewerbsbedingungen bei den Arbeitskosten. Während der Laufzeit der Verträge herrscht Betriebsfrieden. Für Tarifverhandlungen sind die Verbände (Arbeitgeber und Gewerkschaften) zuständig, was die Betriebe entlastet.

Löhne und Arbeitsbedingungen können jedoch nicht nur auf Branchenebene über (Flächen-)Tarifverträge, sondern auch auf Betriebs- oder Unternehmensebene (Firmentarifverträge) oder in individuellen Arbeitsverträgen geregelt werden. Individuelle Arbeitsverträge werden vor allem in kleineren Betrieben geschlossen. Für größere Firmen wird der Verwaltungsaufwand schnell zu groß, wenn mit jedem Beschäftigten einzeln ein Arbeitsvertrag ausgehandelt werden muss. Für diese sind deshalb Firmentarifverträge eine interessante Alternative.

Im Arbeitsrecht haben Tarifverträge Vorrang gegenüber Betriebsvereinbarungen und Einzelarbeitsverträgen und können deshalb auch als Mindestarbeitsbedingungen interpretiert werden. Allerdings gelten Branchentarifverträge ihren Kritikern häufig als zu starr, da sie branchenweit zwischen Gewerkschaften und Arbeitgeberverbänden abgeschlossen werden und somit nicht die Situation jedes einzelnen Betriebs berücksichtigen können. Mittlerweile sind jedoch Öffnungsklauseln in Branchentarifverträgen weit verbreitet, die explizit betriebliche Gestaltungsmöglichkeiten anbieten. Damit haben betriebsbezogene Regelungen und in der Folge auch die betrieblichen Interessenvertretungen, die betriebsspezifische Vereinbarungen aushandeln, an Bedeutung gewonnen.

Der vermuteten „Erosion des Flächentarifvertrags“ und dem Thema „Stärkung der betrieblichen Aushandlungsebene" wird in der Öffentlichkeit unverändert große Aufmerksamkeit entgegengebracht. Weniger wahrgenommen in der öffentlichen Diskussion werden die Betriebe, die formal zwar nicht tarifgebunden sind, sich jedoch freiwillig an einem Branchentarifvertrag orientieren. Um die tatsächliche Bindungswirkung der Flächentarifverträge für die Wirtschaft abschätzen zu können, sollen auch diese Betriebe hier genauer betrachtet werden.

Auf betrieblicher Ebene regelt das Betriebsverfassungsgesetz die institutionalisierte Interessenvertretung der Beschäftigten. Formal herrscht eine klare funktionale Trennung zwischen der Mitbestimmung auf Bran-

Vgl. zuletzt Ellguth/Kohaut 2011, 2010. 
TABELLE 1

\section{Beschäftigte in tarifgebundenen Betrieben 2011 nach Wirtschaftszweig}

Anteile in Prozent

$\begin{array}{cc}\text { Branchentarifvertrag } & \text { Haus-/ } \\ \text { Firmentarifvertrag }\end{array}$

Kein Tarifvertrag

\begin{tabular}{|c|c|c|c|c|c|c|c|c|}
\hline & West & Ost & West & Ost & West & $\begin{array}{c}\text { davon Orientierung am } \\
\text { Branchentarif }\end{array}$ & Ost & $\begin{array}{c}\text { davon Orientierung am } \\
\text { Branchentarif }\end{array}$ \\
\hline Landwirtschaft u. a. & 52 & 15 & 1 & 5 & 47 & 41 & 79 & 38 \\
\hline Energie/Wasser/Abfall u. Bergbau & 71 & 48 & 17 & 30 & 11 & 52 & 22 & 37 \\
\hline Verarbeitendes Gewerbe & 54 & 24 & 10 & 13 & 36 & 58 & 62 & 53 \\
\hline Baugewerbe & 67 & 50 & 3 & 3 & 30 & 63 & 46 & 71 \\
\hline Handel & 43 & 21 & 4 & 6 & 53 & 59 & 73 & 53 \\
\hline Verkehr u. Lagerei & 44 & 25 & 15 & 15 & 41 & 46 & 61 & 45 \\
\hline Information u. Kommunikation & 16 & 11 & 4 & 21 & 79 & 26 & 67 & 44 \\
\hline Finanz- und Versicherungsdienstleistungen & 79 & 51 & 2 & 5 & 18 & 41 & 44 & 44 \\
\hline Gastgewerbe u. Sonst. Dienstleistungen & 45 & 24 & 3 & 7 & 52 & 45 & 68 & 44 \\
\hline Gesundheit u. Erziehung/Unterricht & 57 & 35 & 10 & 20 & 34 & 64 & 45 & 55 \\
\hline Wirtschaftl., wissenschaftl. u. freiberufl. DL & 46 & 47 & 5 & 5 & 49 & 39 & 48 & 39 \\
\hline Organisationen ohne Erwerbscharakter & 57 & 32 & 10 & 12 & 33 & 60 & 56 & 51 \\
\hline Öffentl. Verwaltung/Sozialversicherung & 87 & 83 & 11 & 15 & 2 & 83 & 2 & 57 \\
\hline Gesamt & 54 & 37 & 7 & 12 & 39 & 52 & 51 & 50 \\
\hline
\end{tabular}

chenebene und auf betrieblicher Ebene. Die Praxis ist gleichwohl geprägt von einem vielfältigen Ineinandergreifen beider Ebenen mit wechselseitigen Abhängigkeiten. Betriebsräten obliegt die Umsetzung und Überwachung geltender Tarifverträge.

Seit 1996 erhebt das IAB-Betriebspanel sowohl für West- als auch für Ostdeutschland jährlich Informationen zur Tarifbindung und zur Existenz eines Betriebsrats. 2011 wurden zudem Betriebe, die zwar nicht tarifgebunden sind, sich aber an einem Branchentarifvertrag orientieren, genauer befragt, was diese Orientierung bedeutet. Die aktuellen Ergebnisse beruhen auf Angaben von rund 15.300 Betrieben in beiden Landesteilen. Die Ergebnisse sind repräsentativ für die rund 2,1 Mio. Betriebe mit mindestens einem sozialversicherungspflichtig Beschäftigten. Insgesamt sind in diesen Betrieben etwa 35,2 Mio. Personen beschäftigt.

\section{Ausmaß und Entwicklung der Tarifbindung}

In der folgenden Darstellung wird zwischen der Tarifbindung der Betriebe und der Beschäftigten unterschieden. Betrachtet man zunächst die Beschäftigten, so arbeiten im Jahr 2011 hochgerechnet rund $54 \%$ der westdeutschen und etwa $37 \%$ der ostdeutschen Beschäftigten in einem Betrieb, der einem Branchentarifvertrag unterliegt (Tabelle 1). Firmentarifverträge gelten für $7 \%$ der westdeutschen und $12 \%$ der ostdeutschen Beschäftigten. Für rund $39 \%$ der westdeutschen und $51 \%$ der ostdeutschen Arbeitnehmer gibt es keinen Tarifvertrag. Jeweils rund die Hälfte dieser Arbeitnehmer profitiert jedoch indirekt von Tarifverträgen, da sich ihre Betriebe nach eigenen Angaben daran orientieren. Das Ausmaß der Tarifbindung variiert stark zwischen den Wirtschaftszweigen, während sich die Bedeutung der Tarifbindung in den einzelnen Branchen von Jahr zu Jahr wenig verändert. ${ }^{2}$ Die Tarifbindung liegt bei den Finanz- und Versicherungsdienstleistungen und im Baugewerbe in beiden Landesteilen weit über dem Durchschnitt, während vor allem im Bereich der Information und Kommunikation Branchentarifverträge kaum eine Rolle spielen.

Aufgrund der starken Größenabhängigkeit der Tarifbindung ergibt sich auf Ebene der Betriebe (Tabelle 2), dass hochgerechnet rund $32 \%$ der westdeutschen und nur $18 \%$ der ostdeutschen Betriebe an Branchentarifverträge gebunden sind. Haus- oder Firmentarifverträge gelten für $2 \%$ der Betriebe in den alten und etwa $3 \%$ der Betriebe in den

(2) Seit 2009 erfolgt die Einteilung der Wirtschaftszweige nach der neuen Gliederung des Statistischen Bundesamtes (WZ 2008). Aufgrund dieser Anpassung sind Branchenvergleiche mit den Jahren vor 2009 nicht mehr möglich. 


\section{Tarifbindung der Betriebe 2011 nach Wirtschaftszweig}

Anteile in Prozent

Haus-/

Firmentarifvertrag
Kein Tarifvertrag

\begin{tabular}{|c|c|c|c|c|c}
\hline West & Ost & West & $\begin{array}{c}\text { davon Orientierung } \\
\text { am Branchentarif }\end{array}$ & Ost & $\begin{array}{c}\text { davon Orientierung } \\
\text { am Branchentarif }\end{array}$ \\
\hline 0 & 3 & 63 & 31 & 90 & 33 \\
\hline 6 & 9 & 38 & 38 & 67 & 33 \\
\hline 3 & 4 & 66 & 51 & 83 & 49 \\
\hline 1 & 1 & 46 & 50 & 58 & 63 \\
\hline 2 & 3 & 70 & 49 & 86 & 42 \\
\hline 5 & 8 & 74 & 37 & 85 & 46 \\
\hline 1 & 3 & 92 & 22 & 91 & 37 \\
\hline 1 & 1 & 58 & 36 & 82 & 46 \\
\hline 1 & 4 & 67 & 35 & 84 & 38 \\
\hline 4 & 6 & 60 & 52 & 77 & 59 \\
\hline 1 & 1 & 83 & 33 & 87 & 36 \\
\hline 10 & 4 & 50 & 42 & 74 & 59 \\
\hline 13 & 3 & 6 & 66 & 4 & 32 \\
\hline 2 & 3 & 66 & 42 & 79 & 45 \\
\hline & & & & & \\
\hline
\end{tabular}

Landwirtschaft u. a.

Energie/Wasser/Abfall u. Bergbau

Verarbeitendes Gewerbe

\section{Baugewerbe}

Handel

Verkehr u. Lagerei

Information u. Kommunikation

Finanz- und Versicherungsdienstleistungen

Gastgewerbe u. Sonst. Dienstleistungen

Gesundheit u. Erziehung/Unterricht

Wirtschaftl., wissenschaftl. u. freiberufl. DL

Organisationen ohne Erwerbscharakter

Öffentl. Verwaltung/Sozialversicherung

Gesamt

Branchentarifvertrag

\begin{tabular}{|r|r|}
\hline West & Ost \\
\hline 37 & 8 \\
\hline 56 & 24 \\
\hline 32 & 13 \\
\hline 53 & 42 \\
\hline 28 & 11 \\
\hline 22 & 8 \\
\hline 7 & 6 \\
\hline 41 & 17 \\
\hline 33 & 12 \\
\hline 36 & 18 \\
\hline 16 & 12 \\
\hline 40 & 22 \\
\hline 80 & 93 \\
\hline 32 & 18 \\
\hline
\end{tabular}

Quelle: IAB-Betriebspanel 2011.

TABELLE 3

Tarifbindung der Betriebe 2011 nach Betriebsgröße

Anteile in Prozent

\begin{tabular}{|c|c|c|c|c|c|c|c|c|}
\hline & \multicolumn{2}{|c|}{ Branchentarifvertrag } & \multicolumn{3}{|c|}{$\begin{array}{l}\text { Haus-/ } \\
\text { Firmentarifvertrag }\end{array}$} & \multicolumn{3}{|c|}{ Kein Tarifvertrag } \\
\hline & West & Ost & West & Ost & West & $\begin{array}{l}\text { davon Orientierung } \\
\text { am Branchentarif }\end{array}$ & Ost & $\begin{array}{c}\text { davon Orientierung am } \\
\text { Branchentarif }\end{array}$ \\
\hline 1 bis 9 Beschäftigte & 25 & 13 & 1 & 2 & 73 & 38 & 85 & 44 \\
\hline 10 bis 49 Beschäftigte & 43 & 27 & 3 & 6 & 53 & 55 & 68 & 48 \\
\hline 50 bis 199 Beschäftigte & 53 & 45 & 8 & 12 & 39 & 57 & 43 & 55 \\
\hline 200 bis 499 Beschäftigte & 64 & 51 & 12 & 18 & 24 & 58 & 30 & 47 \\
\hline 500 und mehr Beschäftigte & 76 & 60 & 13 & 29 & 11 & 58 & 11 & 51 \\
\hline Gesamt & 32 & 18 & 2 & 3 & 66 & 42 & 79 & 45 \\
\hline Quelle: IAB-Betriebspanel 2011. & & & & & & & & WSI Mitteilungen \\
\hline
\end{tabular}

neuen Bundesländern. Der Rest, also etwa $66 \%$ der westdeutschen und etwa $79 \%$ der ostdeutschen Betriebe, ist nicht tarifgebunden. Allerdings geben etwa $42 \%$ der nicht tarifgebundenen Betriebe in Westdeutschland und $45 \%$ in Ostdeutschland an, sich in ihren Einzelarbeitsverträgen an bestehenden Branchentarifen zu orientieren.

Betrachtet man die Tarifbindung der Betriebe nach ihrer Größe (Tabelle 3), so zeigt sich wie in den Vorjahren, dass der Deckungsgrad mit zunehmender Beschäftigtenzahl steigt. Das gilt sowohl für die alten als auch für die neuen Bundesländer, wenn auch auf unterschiedlichem Niveau. Während für die Kleinbetriebe die Branchentarifverträge eine untergeordnete Rolle spielen, sind Großbetriebe mit über 500 Beschäftigten in der Mehrheit tarifgebunden. Ebenso nimmt die Bedeutung der Haus- bzw. Firmentarifverträge mit steigender Betriebsgröße zu. 
ABB.

\section{Flächentarifbindung der Beschäftigten 1996-2011}

Anteile in Prozent $\square$ Gesamtwirtschaft West $\square$ Gesamtwirtschaft Ost

- Privatwirtschaft* West Privatwirtschaft* Ost

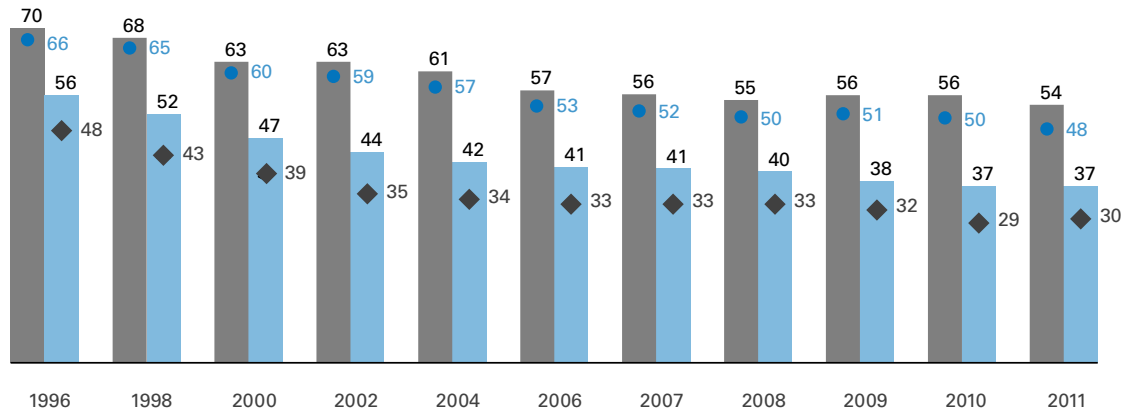

*ohne Landwirtschaft und Organisationen ohne Erwerbszweck.

Anmerkung: Seit 2010 verändertes Editingverfahren, siehe Ellguth/Kohaut 2011, S. 243.

Quelle: IAB-Betriebspanel; Darstellung der Autoren.

ABB. 2

\section{Orientierung am Branchentarifvertrag 2011}

West

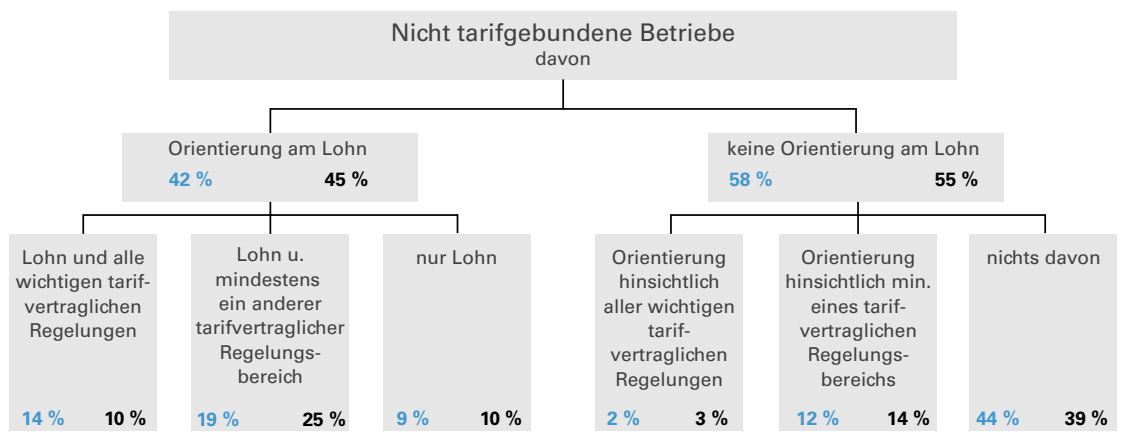

Abweichungen aufgrund von Rundungsfehlern möglich.

Quelle: IAB-Betriebspanel 2011; Darstellung der Autoren.

Seit 1996 bis Mitte der 2000er Jahre war die Branchentarifbindung in den alten wie in den neuen Bundesländern rückläufig. Seither zeichnen sich Stabilisierungstendenzen ab. Aktuell hält die Reichweite der Tarifbindung in Ostdeutschland das Vorjahresniveau. In den westlichen Bundesländern ist nach einer zwischenzeitlichen Konsolidierungsphase für 2011 erneut ein Rückgang der Tarifbindung $\mathrm{zu}$ verzeichnen. $\mathrm{Ob}$ sich daraus ein neuer Abwärtstrend entwickelt, werden die nächsten Jahre zeigen. Abbildung 1 stellt sowohl die gesamtwirtschaftliche Entwicklung als auch die Entwicklung der Branchentarifbindung in der Pri- vatwirtschaft dar. Betrachtet man zunächst die Gesamtwirtschaft in Westdeutschland, so ging die Flächentarifbindung bezogen auf die Beschäftigten von 1996 bis 2011 um 16 Prozentpunkte zurück. In Ostdeutschland belief sich dieser Rückgang auf 19 Prozentpunkte. Nahezu die gleiche Entwicklung ist zu beobachten, wenn nur die Privatwirtschaft betrachtet wird. Das bedeutet aber umgekehrt, dass der Rückgang der Branchentarifbindung durch die Betriebe der Privatwirtschaft getrieben wird, während die Flächentarifbindung im öffentlichen Sektor im betrachten Zeitraum weitgehend stabil blieb.

\section{Orientierung an einem Branchen- tarifvertrag}

Um die über die formalrechtliche Geltung hinausgehende Bedeutung der Branchentarifverträge beurteilen zu können, muss die Gruppe der nicht tarifgebundenen Betriebe, die sich aber an einem Tarifvertrag orientieren, genauer betrachtet werden. Im IAB-Betriebspanel 2011 wurden diese Betriebe - wie auch schon in der Erhebung 2003 - gefragt, ob sie sich hinsichtlich der Löhne und/oder anderer Regelungen wie beispielsweise bei den finanziellen Zusatzleistungen (z. B. Weihnachts-/Urlaubsgeld), den Arbeitszeiten oder der Dauer des Jahresurlaubs nach einem Branchentarifvertrag richten. Bei der ersten Erhebung 2003 (Ellguth/ Kohaut 2005) waren keine größeren Unterschiede zwischen den betreffenden Betrieben in West- und in Ostdeutschland zu beobachten. Damals gaben jeweils rund $43 \%$ der Betriebe ohne Tarifvertrag an, sich bei der Entlohnung ihrer Beschäftigten an einen Branchentarif anzulehnen. Weitere $20 \%$ erklärten, sich hinsichtlich anderer Regelungen am Tarifvertrag zu orientieren.

Abbildung 2 zeigt für das Jahr 2011, in welcher Hinsicht die nicht tarifgebundenen Betriebe Branchentarifverträge als Orientierungshilfe nutzen. $42 \%$ der westdeutschen und $45 \%$ der ostdeutschen Betriebe, die nicht branchentarifgebunden sind, geben an, sich in Bezug auf die Entlohnung an einem solchen zu orientieren.

$14 \%$ der tariffreien Betriebe im Westen und $10 \%$ dieser Betriebe im Osten, die den Branchentarif als Messlatte für die Entlohnung verwenden, orientieren sich darüber hinaus auch noch hinsichtlich aller anderen wichtigen tarifvertraglichen Regelungen an dem entsprechenden Branchentarif. In diesen Betrieben dürften die Arbeitsbedingungen weitgehend mit denen in formalrechtlich tarifgebundenen Unternehmen vergleichbar sein. Noch einmal $19 \%$ der westdeutschen und $25 \%$ der ostdeutschen Betriebe, die sich bei der Entlohnung am Branchentarif orientieren, gewähren ihren Beschäftigten mindestens eine weitere tarifvertragliche Regelung (z. B. finanzielle Zusatzleistung, Urlaubsdauer oder Arbeitszeit). Für $14 \%(2+12)$ der westdeutschen und $17 \%(3+14)$ der ostdeutschen Betriebe ohne Tarifvertrag 
dient der Branchentarif nur hinsichtlich einer oder mehrerer anderer Regelungen als Richtschnur, nicht aber bei der Entlohnung. 2003 waren dies noch $18 \%$ der westdeutschen und $20 \%$ der ostdeutschen Betriebe. Am häufigsten orientieren sich die nicht tarifgebundenen Betriebe hinsichtlich Urlaub (West: $25 \%$, Ost: $33 \%$ ) und Arbeitszeit (West: $21 \%$, Ost: $32 \%$ ) an einem Branchentarifvertrag.

Die Firmen, die sich in Bezug auf die Löhne und Gehälter am Branchentarif orientieren, wurden 2011 zusätzlich gefragt, ob sie im Durchschnitt vergleichbare, höhere oder niedrigere Löhne und Gehälter bezahlen. Etwa 75 \% der westdeutschen und sogar $83 \%$ der ostdeutschen Betriebe geben an, vergleichbare Löhne zu zahlen. Erstaunlich ist, dass $22 \%$ der Betriebe im Westen freiwillig höhere Löhne zahlen als im Branchentarif vorgegeben. Nur $4 \%$ der Firmen im Westen unterschreiten die tarifvertragliche Entlohnung. Im Osten hingegen zahlen nur $8 \%$ der Betriebe, die sich am Branchentarif orientieren, höhere Löhne, während $9 \%$ bei der Entlohnung unterhalb des Tarifvertrags bleiben.

Zusammenfassend lässt sich feststellen, dass bezogen auf alle Betriebe rund $28 \%$ der westdeutschen bzw. $36 \%$ der ostdeutschen Betriebe hinsichtlich der Entlohnung einen Branchentarifvertrag als Richtlinie nehmen. Allerdings orientieren sich nur rund $9 \%$ aller Betriebe im Westen und $8 \%$ im Osten neben dem Lohn auch an allen anderen Regelungen des entsprechenden Branchentarifvertrags. Nur in diesen Betrieben dürften die Beschäftigten Arbeitsbedingungen vorfinden, die denen in branchentarifgebundenen Betrieben weitgehend vergleichbar sind. Folglich werden in insgesamt etwa $40 \%$ der westdeutschen bzw. $27 \%$ der ostdeutschen Firmen die Arbeitsbedingungen direkt oder indirekt durch Branchentarifverträge geregelt. $61 \%$ der Beschäftigten in Westdeutschland und etwa $45 \%$ der Beschäftigten in Ostdeutschland arbeiten in diesen Betrieben. Es zeigt sich also, dass der Branchentarifvertrag immer noch eine wichtige Rolle im deutschen System der Arbeitsbeziehungen spielt, auch wenn sich der prozentuale Anteil der an einen Flächentarifvertrag gebundenen Betriebe und Beschäftigten seit 1996 vermindert hat.

\section{Verbreitung betrieblicher Interessen- vertretungen}

Neben der Tarifautonomie bildet die betriebliche Mitbestimmung die zweite tragende Säule im bundesdeutschen System industrieller Beziehungen. In den bisherigen Veröffentlichungen zur quantitativen Entwicklung der industriellen Beziehungen in Deutschland auf Basis des IAB-Betriebspanels war die betriebliche Ebene im Gegensatz zur sektoralen über Jahre hinweg als Hort weitgehender Stabilität und Kontinuität mit keinen bzw. geringen (inhaltlich nicht interpretierbaren) jährlichen Veränderungen beschrieben worden (siehe z. B. Ellguth 2007). Punktuell muss-

\section{TABELLE 4}

Betriebe bzw. Beschäftigte in Betrieben mit Betriebsrat 1993 bis 2011* Anteile in Prozent

\begin{tabular}{|c|c|c|c|c|}
\hline & \multicolumn{2}{|c|}{ Anteil der Betriebe mit Betriebsrat } & \multicolumn{2}{|c|}{$\begin{array}{l}\text { Anteil der Beschäftigten in } \\
\text { Betrieben mit Betriebsrat }\end{array}$} \\
\hline & $\begin{array}{l}\text { West- } \\
\text { deutschland }\end{array}$ & $\begin{array}{l}\text { Ost- } \\
\text { deutschland }\end{array}$ & $\begin{array}{l}\text { West- } \\
\text { deutschland }\end{array}$ & $\begin{array}{l}\text { Ost- } \\
\text { deutschland }\end{array}$ \\
\hline 1993 & 10 & & 51 & \\
\hline 1996 & 12 & 11 & 51 & 43 \\
\hline 1998 & 10 & 9 & 50 & 40 \\
\hline 2000 & 12 & 12 & 50 & 41 \\
\hline 2001 & 12 & 12 & 50 & 41 \\
\hline 2002 & 11 & 11 & 50 & 42 \\
\hline $2003 * *$ & 11 & 11 & 48 & 40 \\
\hline 2004 & 10 & 9 & 47 & 40 \\
\hline 2005 & 11 & 10 & 47 & 40 \\
\hline 2006 & 10 & 10 & 46 & 39 \\
\hline 2007 & 10 & 10 & 46 & 39 \\
\hline 2008 & 10 & 9 & 45 & 37 \\
\hline 2009 & 10 & 10 & 45 & 38 \\
\hline 2010 & 10 & 10 & 45 & 37 \\
\hline 2011 & 10 & 9 & 44 & 36 \\
\hline
\end{tabular}

*Basis: privatwirtschaftliche Betriebe ab 5 Beschäftigte, ohne Landwirtschaft und Organisationen ohne Erwerbszweck.

**Im Vergleich zum Vorjahr veränderte Fragestellung

Quelle: IAB-Betriebspanel.

te aber auch hier konstatiert werden, dass der Anteil der Beschäftigten, deren Interessen durch einen Betriebsrat vertreten werden, leicht rückläufig ist (Ellguth/Kohaut 2009). Vor diesem Hintergrund soll nun neben der Präsentation aktueller Strukturdaten besonderer Wert auf eine differenzierte Betrachtung der mittlerweile 16 Jahre (Ost) bzw. 19 Jahre (West) zurückreichenden Zeitreihe gelegt werden.

Tabelle 4 weist die Daten zur Verbreitung von Betriebsräten in der Privatwirtschaft (Betriebe ab fünf Beschäftigte) ${ }^{3}$ für Westdeutschland seit 1993 und für die ostdeutschen Bundesländer seit 1996 aus. Für den Anteil der Betriebe mit gesetzlich legitimierter Interessenvertretung bestätigt sich das bisherige Bild. Es lässt sich auch weiterhin weder in den

(3) Die Betrachtung der Betriebe ab fünf Beschäftigte ergibt sich aufgrund der Gesetzeslage, die erst ab dieser Betriebsgröße die Wahl eines Betriebsrats zulässt. Damit sind immerhin rund $43 \%$ der privatwirtschaftlichen Betriebe im Westen und $49 \%$ im Osten per se ohne eine gesetzlich legitimierte Interessenvertretung. Allerdings stellt sich die $\mathrm{Si}-$ tuation weniger dramatisch dar, wenn wir von der Betriebs- zur Beschäftigtenperspektive wechseln und die entsprechenden Anteilswerte betrachten. Da in der großen Anzahl Kleinstbetriebe nur ein relativ kleinerTeil der Gesamtbeschäftigten arbeitet, ergibt sich ein Anteil an Arbeitnehmern, die qua Gesetz ohne einen Betriebsrat bleiben, von $7 \%$ im Westen und $10 \%$ im Osten. 
ABB. 3

\section{Beschäftigte in Betrieben mit Betriebsrat nach Betriebsgröße 1993-2011*}

Anteile in Prozent

- West (501 u.m.) - Ost (501 u.m.) -West (51-500) - Ost (51-500) - West (bis 50) - Ost (bis 50)

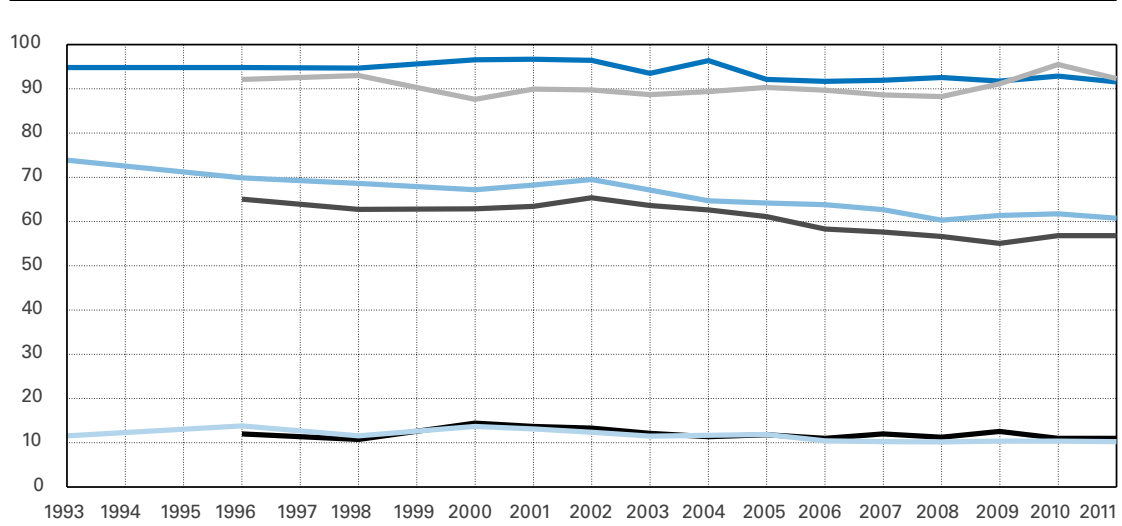

*Basis: privatwirtschaftliche Betriebe ab 5 Beschäftigte ohne Landwirtschaft und Organisationen ohne Erwerbszweck.

Quelle: IAB-Betriebspanel; Darstellung der Autoren.

\section{TABELLE 5}

\section{Verbreitung von Betriebsräten nach Betriebsgröße 2011*}

Anteile in Prozent

\begin{tabular}{|c|c|c|c|c|c|c|}
\hline \multicolumn{7}{|c|}{ Betriebsgrößenklassen (Anzahl der Beschäftigten) } \\
\hline & $5-50$ & $51-100$ & $101-199$ & $200-500$ & 501 u.m. & $\begin{array}{c}\text { Insgesamt } \\
\text { (ab } 5 \text { Besch.) }\end{array}$ \\
\hline \multicolumn{7}{|c|}{ Westdeutschland } \\
\hline Betriebe mit BR & 6 & 38 & 62 & 78 & 88 & 10 \\
\hline Beschäftigte mit BR & 10 & 38 & 63 & 79 & 92 & 44 \\
\hline \multicolumn{7}{|c|}{ Ostdeutschland } \\
\hline Betriebe mit BR & 6 & 37 & 58 & 72 & 92 & 9 \\
\hline Beschäftigte mit BR & 11 & 39 & 59 & 75 & 92 & 36 \\
\hline
\end{tabular}

*Basis: privatwirtschaftliche Betriebe ab 5 Beschäftigte, ohne Landwirtschaft und Organisationen ohne Erwerbszweck.

Quelle: IAB-Betriebspanel 2011
Jahr zu Jahr betrachtet zunächst gering, wobei sich immer wieder Phasen einer (nominellen) Stabilisierung mit solchen geringer Rückgänge abwechseln. In der Gesamtschau ist aber ein klarer Abwärtstrend unverkennbar - auch unter Berücksichtigung der veränderten Fragestellung im IABBetriebspanel seit 2003, durch die die Anteilswerte um ca. einen Prozentpunkt nach unten korrigiert wurden (siehe Ellguth/Kohaut 2005, S. 401). In der aktuellen Befragungswelle ergeben sich für beide Landesteile die geringsten Werte seit Bestehen des IAB-Betriebspanels.

Interessantes fördert nun eine nach Betriebsgröße differenzierte Darstellung zutage (Abbildung 3). Betrachten wir zunächst die Entwicklung in Kleinbetrieben: Bei der Reform des Betriebsverfassungsgesetzes 2001 ging es dem Gesetzgeber ja unter anderem darum, die Erosionsprozesse der betrieblichen Mitbestimmung zu stoppen und mit einer „Erleichterung der Bildung von Betriebsräten durch Entbürokratisierung des Wahlrechts" (Deutscher Bundestag 2001, S. 2) die Neugründung von Betriebsratsgremien in Kleinbetrieben zu forcieren. Unabhängig davon, ob die damals zugrunde liegende Einschätzung einer beständig erodierenden institutionellen Basis, die (ein) Ausgangspunkt für das gesetzgeberische Handeln war, den Tatsachen entsprach, wurde auch in der Folge die quantitative Entwicklung der betrieblichen Mitbestimmung vor allem mit Blick auf die Kleinbetriebe betrachtet. Nach allen vorliegenden Analysen haben sich allerdings die Erwartungen auf einen entsprechenden Gründungsboom von Betriebsratsgremien nicht erfüllt (vgl. Bellmann/Ellguth 2006), wie sich auch mit unserer Zeitreihe für die Betriebe bis 50 Beschäftigte (vgl. Abbildung 3, die unteren zwei Linien) bestätigt.

In Ordnung ist die Welt der betrieblichen Mitbestimmung nach wie vor in den Großbetrieben mit Anteilswerten von über $90 \%$ und einem aktuellen Niveau, das ungefähr dem zu Beginn der Datenerhebung entspricht (vgl. Abbildung 3, die oberen zwei Linien). Von einer Erosion (zumindest in quantitativer Hinsicht) kann hier keine Rede sein. Besondere Aufmerksamkeit verdient aber die Entwicklung in den Betrieben zwischen 51 und 500 Beschäftigten (vgl. Abbildung 3, die zwei mittleren Linien). Der in den Gesamtwerten zu beobachtende Rückgang ist der Entwicklung in diesem Segment geschuldet, in dem ein Betriebsrat weder eine Selbstverständlichkeit noch die Ausnahme ist. Offensichtlich verliert gerade hier die betriebliche Mitbestimmung an Boden. Bemerkenswert ist auch, dass dieser Trend in beiden Landesteilen weitgehend parallel verläuft.

Eine differenzierte Betrachtung der Verbreitung von Betriebsratsgremien nach Betriebsgröße am aktuellen Rand zeigt auch genauer, dass die insgesamt nach wie vor vorhandenen West-Ost-Unterschiede auf die geringere Reichweite der betrieblichen Mitbestimmung im Segment zwischen 101 und 500 Beschäftigten in Ostdeutschland zurückzuführen sind (Tabelle 5).

Ansonsten zeigen sich wiederum die aus den Vorjahren bekannten größenspezifischen Unterschiede. Nur knapp jeder 17. Kleinbetrieb (bis 50 Beschäftigte) verfügt über einen 
Verbreitung von Betriebsräten nach Branche 2011*

Anteile in Prozent

\begin{tabular}{|c|c|c|c|c|c|c|c|c|c|c|c|}
\hline \multicolumn{12}{|c|}{ Wirtschaftszweige } \\
\hline & $\begin{array}{c}\text { Energie/ } \\
\text { Wasser/ } \\
\text { Abfall } \\
\text { Bergbau }\end{array}$ & $\begin{array}{l}\text { Verarb. } \\
\text { Gewerbe }\end{array}$ & $\begin{array}{c}\text { Bau- } \\
\text { gewerbe }\end{array}$ & Handel & $\begin{array}{l}\text { Verkehr/ } \\
\text { Lagerei }\end{array}$ & $\begin{array}{l}\text { Informat./ } \\
\text { Kommunik. }\end{array}$ & $\begin{array}{l}\text { Finanz-/ } \\
\text { Versicher.- } \\
\text { DL }\end{array}$ & $\begin{array}{l}\text { Gastgew. } \\
\text { sonst. DL }\end{array}$ & $\begin{array}{l}\text { Gesundheit, } \\
\text { Erziehung/ } \\
\text { Unterricht }\end{array}$ & $\begin{array}{l}\text { Wirtschaftl., } \\
\text { wiss., frei- } \\
\text { berufl. DL }\end{array}$ & $\begin{array}{c}\text { Insgesamt } \\
\text { (ab } 5 \text { Besch.) }\end{array}$ \\
\hline \multicolumn{12}{|c|}{ Westdeutschland } \\
\hline Betriebe mit BR & 55 & 16 & 3 & 10 & 16 & 14 & 27 & 2 & 10 & 7 & 10 \\
\hline $\begin{array}{l}\text { Beschäftigte } \\
\text { mit BR }\end{array}$ & 86 & 68 & 18 & 31 & 55 & 46 & 69 & 12 & 42 & 30 & 44 \\
\hline \multicolumn{12}{|c|}{ Ostdeutschland } \\
\hline Betriebe mit BR & 18 & 12 & 3 & 9 & 10 & 8 & 28 & 7 & 14 & 10 & 9 \\
\hline $\begin{array}{l}\text { Beschäftigte } \\
\text { mit BR }\end{array}$ & 65 & 48 & 13 & 23 & 34 & 21 & 72 & 16 & 53 & 33 & 36 \\
\hline
\end{tabular}

*Basis: privatwirtschaftliche Betriebe ab 5 Beschäftigte, ohne Landwirtschaft und Organisationen ohne Erwerbszweck.

Quelle: IAB-Betriebspanel 2011.

Betriebsrat. Ab 200 Beschäftigte (Freistellungsgrenze) sind es dann $80 \%$ im Westen und $70 \%$ im Osten. Prägt in betrieblicher Perspektive die große Zahl der Kleinbetriebe das Gesamtbild, so relativiert sich deren Gewicht natürlich bei Betrachtung des auf Beschäftigte bezogenen Deckungsgrads.

Auch in der veränderten Wirtschaftszweigsystematik bestätigen sich die erwarteten starken Unterschiede im Deckungsgrad in einzelnen Branchen, und das sowohl mit Blick auf die Anzahl der Betriebe als auch der Beschäftigten mit Betriebsrat (Tabelle 6). Die größte quantitative Reichweite der betrieblichen Mitbestimmung besteht im traditionell stark mitbestimmten Bereich Energie- und Wasserversorgung/Abfallwirtschaft/Bergbau, in den Finanz- und Versicherungsdienstleistungen, im Verarbeitenden Gewerbe und im Bereich Verkehr/Lagerei. Das untere Ende bilden die vorwiegend kleinbetrieblich strukturierten Branchen des sonstigen Dienstleitungsbereichs (inkl. Gastgewerbe) und der Bauwirtschaft. Branchenspezifische Entwicklungen lassen sich leider - wie schon erwähnt - wegen der 2009 erfolgten Umstellung auf eine neue Wirtschaftszweigsystematik (WZ2008) nicht verfolgen. Hier müssen noch einige Erhebungswellen des IAB-Betriebspanels folgen, bevor Branchentrends identifiziert werden können.

\section{Das Zusammenspiel von betrieb- licher und überbetrieblicher Interessenvertretung}

Auf das eigentümliche Ineinandergreifen der beiden formal unabhängigen Institutionen des bundesdeutschen Systems industrieller Beziehungen - Tarifautonomie und betriebliche Mitbestimmung - mit ihren wechselseitigen Abhängigkeiten und die daraus resultierende Fähigkeit zur kooperativen Konfliktverarbeitung wurde bereits hingewiesen (z. B. Schmidt/Trinczek 1997; Müller-Jentsch 2003). Das Verhältnis von tarifvertraglicher und betrieblicher Regulierung bleibt auch angesichts der immer wieder, nicht nur zu Wahlkampfzeiten aufflammenden politischen Diskussion um die Zukunft des dualen Systems virulent.

Der abschließende und vergleichende Blick auf die betriebliche und sektorale Ebene der (gesetzlich legitimierten) Interessenvertretung soll einen Eindruck von den Verschiebungen im dualen System ermöglichen. Damit gilt im Folgenden das Augenmerk zum einen der Entwicklung der sogenannten betrieblichen Vertretungslücken, also Betrieben, die zwar tarifgebunden sind, aber über keinen Betriebsrat verfügen; zum anderen geht es um die sogenannten weißen Flecken in der Tarif- und Mitbestimmungslandschaft, wo es keinerlei gesetzlich legitimierte Interessenvertretung gibt. Die präsentierten Zahlen beziehen sich ausschließlich auf die Betriebe der Privatwirtschaft ab fünf Beschäftigte. Eine Berücksichtigung der Kleinstbetriebe, die ja nicht zur Wahl eines Betriebsrats berechtigt sind, würde bei der 
TABELLE 7

\section{Tarifbindung und Betriebsrat 2011*}

Anteil der Beschäftigten in Prozent

\begin{tabular}{|c|c|c|c|c|c|c|c|c|}
\hline & \multicolumn{4}{|c|}{ Westdeutschland } & \multicolumn{4}{|c|}{ Ostdeutschland } \\
\hline & \multirow{2}{*}{$\begin{array}{c}\text { Privat- } \\
\text { wirtschaft }\end{array}$} & \multicolumn{3}{|c|}{ darunter: } & \multirow{2}{*}{$\begin{array}{c}\text { Privat- } \\
\text { wirtschaft }\end{array}$} & \multicolumn{3}{|c|}{ darunter: } \\
\hline & & $\begin{array}{l}\text { Verarb. } \\
\text { Gewerbe }\end{array}$ & Bauwirtschaft & $\begin{array}{l}\text { Dienst- } \\
\text { leistungen }\end{array}$ & & $\begin{array}{l}\text { Verarb. } \\
\text { Gewerbe }\end{array}$ & Bauwirtschaft & $\begin{array}{l}\text { Dienst- } \\
\text { leistungen }\end{array}$ \\
\hline BR und Branchentarif & 29 & 46 & 14 & 23 & 17 & 20 & 8 & 18 \\
\hline BR und Haustarif & 6 & 10 & 2 & 5 & 10 & 11 & 2 & 12 \\
\hline BR und kein Tarif & 8 & 13 & 1 & 5 & 9 & 16 & 2 & 9 \\
\hline Branchentarif und kein BR & 21 & 9 & 57 & 26 & 15 & 5 & 44 & 17 \\
\hline Haustarif und kein BR & 1 & 0 & 1 & 2 & 2 & 2 & 1 & 3 \\
\hline kein Tarif und kein BR & 34 & 22 & 25 & 39 & 47 & 45 & 42 & 41 \\
\hline Gesamt & 100 & 100 & 100 & 100 & 100 & 100 & 100 & 100 \\
\hline $\begin{array}{l}\text { Beschäftigte in Betrieben mit } \\
\text { Branchentarifvertrag }\end{array}$ & 50 & 55 & 71 & 49 & 32 & 25 & 53 & 35 \\
\hline $\begin{array}{l}\text { Beschäftigte in Betrieben mit } \\
\text { Betriebsrat }\end{array}$ & 44 & 68 & 18 & 33 & 36 & 48 & 13 & 39 \\
\hline
\end{tabular}

gemeinsamen Betrachtung des Deckungsgrades von betrieblicher Mitbestimmung und Tarifbindung ein systematisch verzerrtes Bild liefern.

Die aktuellen Zahlen bestätigen das bekannte Bild: Nur eine Minderheit der in der Privatwirtschaft beschäftigten Arbeitnehmer sind in Betrieben (ab fünf Beschäftigte) tätig, die zur Kernzone des dualen Systems der Interessenvertretung gehören. Diese Zone umfasst ein knappes Drittel (29\%) der Beschäftigten in Westdeutschland und gerade noch ein Sechstel (17\%) in Ostdeutschland (Tabelle 7). Bemerkenswert ist die mit $10 \%$ relativ große Bedeutung von Betrieben mit Haustarifen und einem Betriebsrat in den neuen Bundesländern. Von einer betrieblichen Vertretungslücke (Betrieb ist an einen Branchentarif gebunden, verfügt aber über keinen Betriebsrat) sind $21 \%$ der Beschäftigten in Westdeutschland, aber nur $15 \%$ in Ostdeutschland betroffen. Diese vermeintlich günstigere Situation in den neuen Bundesländern ist allerdings nur der dort geringeren Reichweite der Tarifbindung geschuldet, die sich in deutlicher ausgeprägten weißen Flecken der Tarif- und Mitbestimmungslandschaft äußert. Während in Westdeutschland „nur“ 34 \% der Beschäftigten ohne Branchentarif und ohne Betriebsrat auskommen müssen, sind dies in Ostdeutschland $47 \%$.

Um das Bild etwas zu erweitern und mögliche branchenspezifische Unterschiede hinsichtlich der beschriebenen „Problemzonen“ zu identifizieren, sind die Ergebnisse zur Reichweite von Tarifverträgen und betrieblicher Mitbestim- mung in Tabelle 7 zusätzlich für das Verarbeitende Gewerbe, die Bauwirtschaft und den Dienstleistungsbereich ${ }^{\oplus}$ dargestellt. Für Westdeutschland lässt sich feststellen, dass die Kernzone des dualen Systems im Verarbeitenden Gewerbe knapp die Hälfte der Beschäftigten (46\%) umfasst und damit weit über den Durchschnitt der Privatwirtschaft hinausreicht. Mit nur jedem siebten bzw. vierten von Tarifbindung und betrieblicher Interessenvertretung erfassten Arbeitnehmer fallen dagegen insbesondere die Bauwirtschaft und auch der Dienstleistungsbereich deutlich ab.

Im Baugewerbe haben vor allem die betrieblichen Vertretungslücken (57 \%) einen ganz beträchtlichen Umfang. Die deutlich bessere Reichweite von Branchentarifverträgen dürfte wohl in erster Linie den dort geltenden Allgemeinverbindlichkeitsregelungen geschuldet sein. Der Dienstleistungsbereich tut sich hingegen mit ausgedehnten „weißen Flecken“ (39 \%) hervor. Im Verarbeitenden Gewerbe bleiben diese „Problemzonen“ dagegen in relativ engen Grenzen.

In Ostdeutschland ergibt sich ein etwas anderes Bild. Hier unterscheidet sich das Verarbeitende Gewerbe weit weniger von der restlichen Privatwirtschaft als das in den

(4) Der Dienstleistungsbereich in Tabelle 7 umfasst die Finanzund Versicherungsdienstleistungen, das Gastgewerbe, die Sonstigen Dienstleistungen, das Gesundheitswesen, den Bereich Erziehung und Unterricht und die wirtschaftlichen, wissenschaftlichen und freiberuflichen Dienstleistungen. 
westlichen Bundesländern der Fall ist. Die Kernzone der Interessenvertretung ist mit $20 \%$ kaum größer als im Dienstleistungsbereich (18 \%) und fällt im Vergleich zum Verarbeitenden Gewerbe Westdeutschlands (46\%) stark ab. Offensichtlich ist in Ostdeutschland der industrielle Kernbereich nicht im gleichen überdurchschnittlichen Maße mit den beiden Institutionen der Interessenvertretung ausgestattet, wie das traditionell im Westen der Fall ist. Für den Dienstleistungsbereich ergibt sich hingegen in den neuen Bundesländern ein in Relation weit weniger negatives Bild als in Westdeutschland.

\section{Resümee}

Auch wenn die Entwicklung der Branchentarifbindung am aktuellen Rand nicht einheitlich verläuft, so ist in der langen Sicht die rückläufige Tendenz eindeutig. Interessanterweise ist dabei eine Angleichung zwischen Ost- und Westdeutschland nicht zu erkennen. Offensichtlich sind in beiden Landesteilen die gleichen Mechanismen parallel am Werk, die die Entwicklung der Branchentarifbindung bestimmen. Eine angemessene Würdigung der Reichweite bzw. Ausstrahlung tarifvertraglicher Standards bedarf allerdings der Berücksichtigung derjenigen Betriebe, die sich in der Regulierung ihrer Arbeitsbedingungen am Branchentarif orientieren. Eine differenzierte Betrachtung zeigt, dass ein nicht zu vernachlässigender Teil der Beschäftigten auch ohne formelle Tarifbindung ihrer Betriebe Arbeitsbedingungen vorfindet, die tariflichen Standards entsprechen. Allerdings gibt es einen deutlich größeren Teil an Betrieben, die sich nur hinsichtlich einzelner Regelungsbereiche an einem Branchentarif orientieren, sodass die dortigen Arbeitsbedingungen nicht insgesamt den tarifvertraglichen Standard erreichen.

Auch die Reichweite der betrieblichen Mitbestimmung entwickelt sich seit 1996 in beiden Landesteilen weitgehend parallel, wenn auch auf unterschiedlichem Niveau. Hier bleibt abzuwarten, ob sich der durch die Entwicklung im Betriebsgrößensegment zwischen 51 und 500 Beschäftigten getriebene negative Trend in den nächsten Jahren fortsetzt und ob diese Entwicklung in Ost- und Westdeutschland weiterhin im Gleichschritt verlaufen wird.

\section{LITERATUR}

Bellmann, L./Ellguth, P. (2006): Verbreitung von Betriebsräten und ihr Einfluss auf die betriebliche Weiterbildung, in: Jahrbücher für Nationalökonomie und Statistik 226 (5), S. 487-504

Deutscher Bundestag (2001): Entwurf eines Gesetzes zur Reform des Betriebsverfassungsgesetzes, Drucksache 14/5741, Berlin, http://dipbt.bundestag.de/ dip21/btd/14/057/1405741.pdf

Ellguth, P. (2007): Betriebliche und überbetriebliche Interessenvertretung - Ergebnisse aus dem IAB-Betriebspanel 2005, in: WSI-Mitteilungen 60 (3),

S. $155-157$

Ellguth, P./Kohaut, S. (2005): Tarifbindung und betriebliche Interessenvertretung: Aktuelle Ergebnisse aus dem IAB-Betriebspanel, in: WSI-Mitteilungen 58 (7), S. 398-403, http://www.boeckler.de/wsimit_2005_07_ellguth.pdf

Ellguth, P./Kohaut, S. (2009): Tarifbindung und betriebliche Interessensvertretung in Ost und West, in: IAB-Forum 2/2009, S. 68-74

Ellguth, P./Kohaut, S. (2010): Tarifbindung und betriebliche Interessenvertretung: Aktuelle Ergebnisse aus dem IAB-Betriebspanel 2009, in: WSI-Mitteilungen 63 (4), S. 204-209, http://www.boeckler.de/wsimit_2010_04_ellguth.pdf Ellguth, P./Kohaut, S. (2011): Tarifbindung und betriebliche Interessenvertretung: Aktuelle Ergebnisse aus dem IAB-Betriebspanel 2010, in: WSI-Mitteilungen 64 (5), S. 242-247, http://www.boeckler.de/wsimit_2011_05_ellguth.pdf Müller-Jentsch, W. (2003): Re-assessing Co-determination, in: Müller-Jentsch, W./Weitbrecht, H. (Hrsg.): The Changing Contours of German Industrial Relations, München/Mering, S. 39-56

Schmidt, R./Trinczek, R. (1991): Duales System: Tarifliche und betriebliche Interessenvertretung, in: Müller-Jentsch, W. (Hrsg.): Konfliktpartnerschaft. Akteure und Institutionen der industriellen Beziehungen, München/Mering, S. 167-199

\section{AUTOREN}

PETER ELLGUTH, wissenschaftlicher Mitarbeiter im Institut für Arbeitsmarktund Berufsforschung (IAB) der Bundesagentur für Arbeit im Forschungsbereich „Betriebe und Beschäftigung”. Arbeitsschwerpunkte: Arbeitsbeziehungen und betriebliche Arbeitszeitpolitik.

peter.ellguth@iab.de

SUSANNE KOHAUT, Dr., wissenschaftliche Mitarbeiterin im IAB im Forschungsbereich „Betriebe und Beschäftigung“. Arbeitsschwerpunkte: Arbeitsbeziehungen und Betriebsgründungen.

susanne.kohaut@iab.de 